\title{
Avaliação de programas de atenção pós- parto no Brasil: perfil bibliométrico da produção científica (2000-2019)
}

\author{
Evaluation of postpartum programs in Brazil: bibliometric profile of \\ scientific production (2000-2019)
}

Ana Maria Bourguignon', Zulmira Hartz², Dirceia Moreira'

DOI: $10.1590 / 0103-1104202113026$

RESUMO A assistência puerperal constitui um componente da atenção à saúde das mulheres, contemplado em programas desenvolvidos no Brasil, que visam à melhoria dos indicadores de saúde materno-infantil. O estudo objetivou analisar o estado da arte dos estudos avaliativos sobre programas relacionados à atenção puerperal no Brasil, no período de 2000 a 2019. A pesquisa limitou-se à identificação e análise de artigos publicados em periódicos revisados por pares. A revisão foi realizada a partir de pesquisa dos descritores Avaliação, Programas e pós-parto nos portais da BVS, SciELO e Scopus, complementada pela verificação das referências citadas nos artigos para inclusão de estudos pertinentes. Ao total, foram identificados 42 artigos, os quais foram analisados segundo critérios previamente estabelecidos. Os resultados do perfil bibliométrico da produção intelectual identificaram: ausência de autores/centros de pesquisa especializados na interface Avaliação e Programas de atenção pós-parto, disparidades regionais na produção do conhecimento, maior quantidade de artigos sobre o Programa de Humanização no Pré-Natal e Nascimento e a Iniciativa Hospital Amigo da Criança, escassa utilização de referências teórico-metodológicas da área de avaliação. A discussão realizada procura contextualizar a produção científica analisada em relação à constituição do espaço da avaliação em saúde no Brasil.

PALAVRAS-CHAVE Avaliação em saúde. Saúde da mulher. Período pós-parto. Bibliometria.

ABSTRACT Postpartum care is a component of women's health care, included in programs developed in Brazil, with the objective of improving maternal and child health indicators. The study aimed to analyze the state of the art of evaluative studies on programs related to puerperal care in Brazil from 2000 to 2019. The research was limited to the identification and analysis of articles published in peer-reviewed journals. The review was carried out based on research of the descriptors evaluation, programs and postpartum in the $B V S, S c i E L O$ and Scopus portals, complemented by the verification of the references cited in the articles to include relevant studies. In total, 42 articles were identified, which were analyzed according to previously established criteria. The results of the bibliometric profile of intellectual production identified: absence of authors/research centers specialized in the evaluation interface and postpartum care programs, regional disparities in the production of knowledge, more articles on the Prenatal and Birth Humanization Program and the Baby Friendly Hospital Initiative, with scarce use of theoretical and methodological references in

1 Universidade Estadual de Ponta Grossa (UEPG) Ponta Grossa (PR), Brasil. anamariabourg@gmail.com

2 Instituto de Higiene e Medicina Tropical (IHMT)

- Lisboa, Portugal. the field of Evaluation. The discussion carried out seeks to contextualize the scientific production analyzed in relation to the constitution of the space of health Evaluation in Brazil.

KEYWORDS Health evaluation. Women's health. Postpartum period. Bibliometrics. 


\section{Introdução}

A constituição e o desenvolvimento do espaço da avaliação em saúde no Brasil decorrem de três processos sociais concomitantes: a criação do Sistema Único de Saúde (SUS) - que, ao demandar a elaboração e a implantação de políticas para o setor, despertou o interesse pela avaliação dos efeitos das ações governamentais realizadas; a formação da área de saúde coletiva como espaço aglutinador de pesquisadores e gestores de saúde; os financiamentos obtidos junto a organismos internacionais que exigiam, como contrapartida, a avaliação de intervenções, sobretudo na atenção básica'1. Assim, nos anos 1990, houve não só um processo para a institucionalização de práticas avaliativas no SUS - a exemplo do programa Reforço e Organização do SUS (Reforsus) criado, em 1996, para fomentar a qualidade da assistência e da gestão - como um crescimento do interesse acadêmico pela avaliação em saúde'

Para Furtado e Vieira-da-Silva', o espaço da avaliação em saúde no Brasil desenvolve-se no entrelaçamento entre o 'campo burocrático', representado pela esfera governamental, e o 'campo científico', constituído pelas universidades. A partir do ano 2000, os autores identificaram um aumento significativo do número de artigos publicados em periódicos nacionais e de comunicações em eventos científicos. A busca por autonomia da avaliação frente a outras subáreas da saúde coletiva pode ser ilustrada pela criação, no ano de 2006, do 'Grupo Temático de Monitoramento e Avaliação de Programas, Serviços, Sistemas e Políticas de Saúde' - GT de Avaliação da Associação Brasileira de Saúde Coletiva (Abrasco).

A pesquisa bibliométrica elaborada por Furtado et al. ${ }^{2}$ sobre a produção na área de saúde coletiva no período de 1980 a 2016, confirma a tendência de crescimento da avaliação em saúde depois dos anos 2000, em comparação com a subárea de planejamento. Embora o planejamento e avaliação integrem etapas do ciclo de políticas públicas, no âmbito do planejamento, os estudos teóricos e metodológicos têm como foco a análise de situações-problema e a elaboração de intervenções - tais como planos, programas, projetos - para os sistemas e serviços de saúde. Na subárea da avaliação, as investigações realizadas dedicam-se a julgar o valor de uma intervenção ou de um de seus componentes, a fim de compreender as relações entre recursos, processos e resultados, no sentido de identificar de que modo a intervenção elaborada altera a situação-problema que a demandou. Estudos com este enfoque expandiram-se a partir dos anos 2000, inclusive com o aumento do número de grupos de pesquisa de avaliação, fenômeno que coincidiu com a institucionalização de práticas avaliativas no $\mathrm{SUS}^{3}$.

Esse contexto também foi favorável à ampliação dos estudos dedicados à saúde materna e infantil, especialmente, sobre o modelo de humanização da assistência obstétrica e neonatal. O debate sobre as condições de gestar, parir e nascer no Brasil, envolveu um conjunto de fatores, dentre os quais, destacam-se: organização e fortalecimento de movimentos sociais de direitos das mulheres, reconhecimento dos direitos sexuais e reprodutivos como direitos humanos no âmbito de conferências internacionais, recomendações de organizações supranacionais para assistência obstétrica e neonatal baseadas em evidências, elaboração de programas de saúde e ações governamentais voltadas à redução da morbimortalidade materna e neonatal $\mathbf{4}^{4,5}$.

Especialmente a partir dos anos 2000, o Ministério da Saúde elaborou uma série de intervenções direcionadas à melhoria da atenção obstétrica e neonatal, como o Programa de Humanização no Pré-Natal e Nascimento - PHPN (2000), o Pacto pela Redução da Mortalidade Materna e Neonatal (2004) e a Rede Cegonha (2011). À medida que foram organizadas intervenções pelo governo federal, em parceria com estados e municípios, para melhoria dos indicadores de saúde materna e infantil, a produção científica nacional sobre a temática também se ampliou significativamente. Dados relativos à produção de teses e dissertações em programas de pós-graduação brasileiros sobre humanização do parto e nascimento, no período de 1987 a 2012, mostram que $96 \%$ de 
um total de 379 trabalhos foram apresentados a partir do ano 2000, com pico no ano de $2012^{5}$.

$\mathrm{O}$ aumento quantitativo de trabalhos científicos, a partir da primeira década do século XXI, também está atrelado à expansão da pós-graduação stricto sensu, principalmente por meio de políticas indutoras geridas pela Coordenação de Aperfeiçoamento de Pessoal de Nível Superior (Capes), pelo Conselho Nacional de Desenvolvimento Científico e Tecnológico (CNPq) e fundações estaduais de amparo à pesquisa, em parceria com as universidades. Conforme Nobre e Freitas ${ }^{6}$, enquanto no ano de 1990 registravam-se 1.462 programas de pós-graduação, incluindo mestrados e doutorados, no ano de 2016 havia 6.131 programas. A produção científica nacional desenvolveu-se historicamente centrada nos programas de pós-graduação vinculados às universidades 7 .

Ainda que evidenciada a expansão da produção científica, o estudo das teses e dissertações sobre humanização da assistência obstétrica e neonatal também concluiu que o parto e o pré-natal foram mais estudados do que o período puerperal ${ }^{8}$. O pós-parto ou puerpério é definido como o período que se inicia com a dequitação da placenta e que termina quando a mulher volta a menstruar, o que pode variar de mulher para mulher e cuja duração depende diretamente da prática da amamentação. Contudo, as mudanças que afetam as puérperas envolvem, além das alterações de ordem biológica, transformações intensas de caráter psicológico e social ${ }^{9}$. Assim, as necessidades de saúde das puérperas são multidimensionais e influenciadas pela construção social da maternidade.

A assistência após o parto constitui um componente da atenção integral à saúde das mulheres ${ }^{\mathbf{1 0}}$, configurada por meio de ações como a consulta puerperal, apoio à amamentação, orientação quanto ao planejamento reprodutivo e visita domiciliar na primeira semana pós-parto. $\mathrm{O}$ Ministério da Saúde do Brasil recomenda que os serviços de saúde orientem às gestantes sobre o retorno para consulta puerperal - também denominada consulta de revisão do parto - de sete a dez dias pós-parto"1. Também preconiza que pelo menos uma consulta puerperal aconteça nas primeiras semanas do nascimento, a fim de que um profissional habilitado avalie tanto os aspectos clínico-ginecológicos quanto o estado emocional e social em que se encontra a puérpera ${ }^{12}$.

Pesquisas, entretanto, indicam que há falhas e desigualdades na cobertura da atenção puerperal, bem como dificuldades dos serviços em reconhecer as necessidades de saúde das puérperas. Abordagens qualitativas sobre as percepções das puérperas em relação aos cuidados profissionais após o parto, mostram que a atenção centrada no bebê ocorre em detrimento do acolhimento às necessidades de saúde das mulheres ${ }^{\mathbf{1 3}, \mathbf{1 4}}$. De acordo com a Organização Mundial da Saúde (OMS) ${ }^{\mathbf{1 5}}$, o período puerperal recebe menos atenção especializada se comparado à gestação e parto. A pesquisa de Langlois et al. ${ }^{16}$ concluiu, ainda, que as desigualdades quanto ao uso dos serviços de cuidados pós-parto, nos países de baixa e média renda, devem-se a fatores relacionados ao nível educacional, status socioeconômico e dificuldades de acesso das mulheres aos estabelecimentos de saúde.

Diante do panorama exposto, o presente artigo teve como objetivo analisar o estado da arte, na área de avaliação, sobre a atenção puerperal no Brasil, no período de 2000 a 2019. Para tanto, delineamos o perfil bibliométrico dos estudos publicados contextualizando a produção científica analisada em relação à constituição do espaço da avaliação em saúde no Brasil.

\section{Metodologia}

O presente estudo adaptou a metodologia de scoping review, proposta por Arksey e O'Malley ${ }^{17}$, para a revisão dos estudos avaliativos sobre programas desenvolvidos no Brasil, relacionados à prestação de cuidados de saúde no período pós-parto. O desenvolvimento da pesquisa compreendeu seis etapas: elaboração das perguntas de pesquisa; definição dos descritores e critérios de busca; seleção dos estudos nas bases de indexação de periódicos acadêmicos; extração e organização 
dos dados em planilhas e fichas; verificação das referências citadas para inclusão de estudos pertinentes não selecionados anteriormente; síntese e relatório da pesquisa.

A revisão da literatura ora apresentada procurou responder a seguinte questão: Quais são e como se caracterizam os estudos que avaliam programas relacionados à atenção pós-parto no Brasil? Como estratégia metodológica para cobrir o maior número possível de estudos, a busca pelos descritores - cadastrados no vocabulário controlado da terminologia em ciências da saúde presente no Portal da Biblioteca Virtual em Saúde (BVS) - ocorreu em quatro idiomas e com a combinação dos operadores 'and' e 'or' na seguinte disposição: "(avaliação and 'pós-parto' and programa) or (evaluation and postpartum and program) or (evaluación and posparto and programa) or (évaluation and 'post-partum' and programme)". As pesquisas ocorreram no mês de janeiro de 2020, nas bases de indexação de artigos científicos da BVS, SciELO - Scientific Eletronic Library Online e Scopus.

Previamente, foram delineados critérios para inclusão dos textos: Brasil como assunto; texto completo disponível; intervalo de ano de publicação de 2000 a 2019; tipo de documento restrito a artigo revisado por pares; idiomas do texto em português, inglês, espanhol ou francês. Foram excluídos textos que não abordaram o período pós-parto da mulher, bem como aqueles que não realizaram avaliação de programa ou de outra intervenção em saúde. Assim, não foram selecionadas as pesquisas realizadas com puérperas, mas com interesse no pré-natal e no parto. Também não foram incluídos os estudos sobre problemas associados ao puerpério e que não avaliaram programas ou práticas de atenção à saúde, a exemplo dos diversos trabalhos sobre prevalência da depressão pós-parto.

A busca na BVS, biblioteca que possibilita o acesso a várias bases científicas, resultou na seleção de 13 artigos, dos quais 10 constavam vinculados à coleção da Literatura Latino-Americana e do Caribe em Ciências da Saúde (Lilacs) e três à base Medline. Por sua vez, as pesquisas nos portais SciELO e Scopus resultaram na identificação de sete e 15 artigos, respectivamente. As listas de cada base foram comparadas para checar as duplicações, perfazendo-se, assim, 28 estudos. Para determinar o corpus de análise, foi realizada uma etapa complementar, com a leitura dos títulos das referências citadas nos artigos selecionados. Esta etapa acrescentou 14 trabalhos, o que gerou um total de 42 estudos analisados. A síntese de todo o processo encontra-se na figura 1.

Figura 1. Síntese do processo de composição do corpus de análise

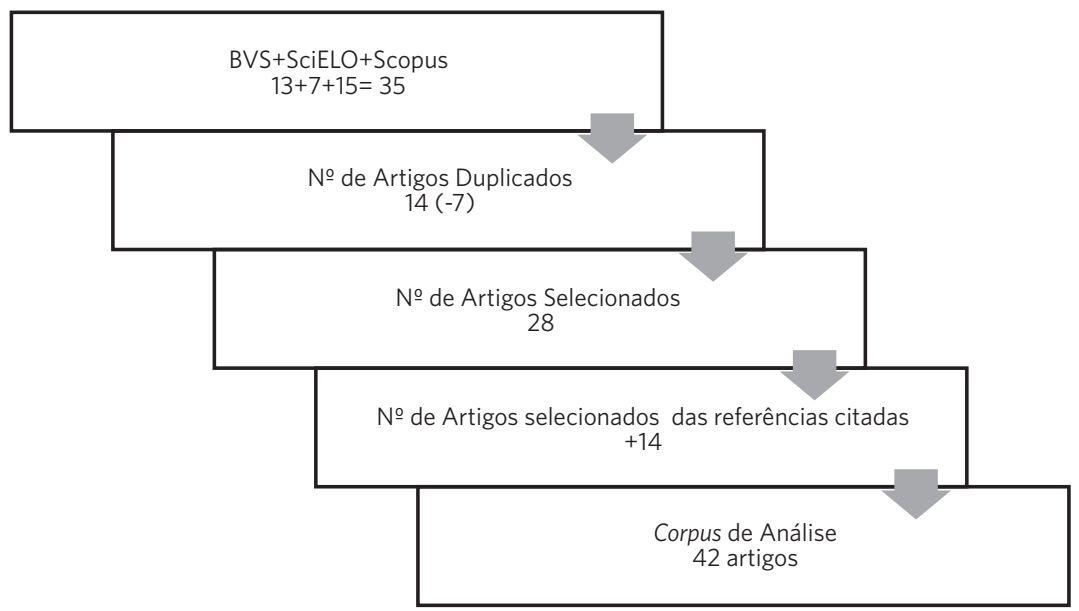


Para a coleta e organização dos dados extraídos dos textos, foi construída uma planilha com base em um roteiro previamente elaborado, contendo as seguintes categorias de análise: a) aspectos gerais de identificação do estudo: título, autores, instituições envolvidas, palavras-chave, ano de publicação, periódico, base de indexação do artigo; b) características do estudo: programa objeto do estudo, unidade territorial do programa avaliado, método e técnicas de pesquisa, documentos e referências citadas. Os dados coletados possibilitaram caracterizar o perfil bibliométrico da produção intelectual.

A revisão bibliométrica constitui uma técnica de análise quantitativa da literatura, que procura medir a produção e a difusão do conhecimento científico. A técnica é útil para mapear e acompanhar o desenvolvimento de um determinado campo ou temática de estudos, verificar o volume e a distribuição geográfica e institucional dos trabalhos, padrões de autoria e publicação, usos dos resultados, bem como outros elementos atinentes ao interesse da pesquisa. Os trabalhos de revisão da literatura podem utilizar os dados bibliométricos para contextualizar os aspectos sócio-históricos da produção científica ${ }^{\mathbf{1 8 - 2 0}}$.
Para a análise das palavras-chave dos artigos, utilizou-se o software Iramuteq (Interface de R pour les Analyses Multidimensionalles de Textes et de Questionnaires, Laboratorie Lerass, Université de Toulouse), criado por Pierre Ratinaud. De acordo com Camargo e Justo ${ }^{21}$, o programa gratuito é uma ferramenta útil para organizar o vocabulário em formatos visualmente claros. A nuvem de palavras é um recurso que considera a frequência das palavras para distribuí-las em um esquema visual, enquanto a análise de similitude indica as conexões entre as palavras. Ambos os recursos foram utilizados para apresentar os resultados do mapeamento e análise da produção científica sobre avaliação de programas de atenção pós-parto no Brasil.

\section{Resultados}

Em vinte anos, o número de artigos encontrados pode ser considerado baixo. As duas primeiras publicações do período datam de 2003 e ambas realizaram avaliações da Iniciativa Hospital Amigo da Criança ${ }^{22,23}$. No gráfico 1, observa-se a distribuição temporal dos estudos, havendo maior concentração no período de 2010 a 2015, com 17 artigos.

Gráfico 1. Distribuição temporal dos estudos avaliativos de programas sobre atenção pós-parto no Brasil (2000-2019)

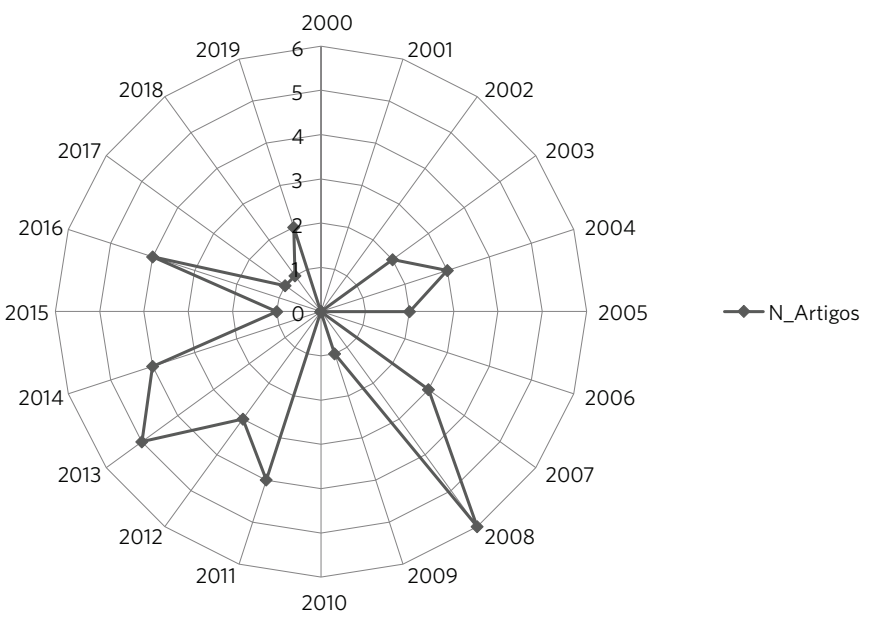


Os 42 artigos estão publicados em 30 diferentes periódicos, majoritariamente nacionais. As seguintes revistas publicaram mais de um artigo: 'Cadernos de Saúde Pública' (4) 24-28, 'Revista Brasileira de Saúde Materno Infantil' (4) 23,28-30, 'Jornal de Pediatria' (3) ${ }^{31-33}$, 'Revista de Saúde Pública' (3) 34-36, 'Escola Anna Nery Revista de Enfermagem' (2) 37,38, 'Pan American Journal of Public Health' (2) ${ }^{39,40}$. Esta última, juntamente com 'American Journal of Public Health'22, 'Journal of Human Lactation'41 e a 'Scientific World Journal'42, compõem os quatro periódicos internacionais onde foram veiculados artigos analisados. Todas as revistas estão classificadas no Qualis Periódicos da Capes (quadriênio 2013-2016), distribuídas nos seguintes estratos: A1 - 1, A2 -10, B1 - 17, B2 - 9, B3-3, B4-2. Das 26 revistas nacionais, 16 estão ranqueadas entre as cem publicações principais de língua portuguesa, do Google Scholar. Embora a busca pelos artigos tenha abrangido descritores em quatro idiomas distintos, foram identificados estudos publicados em português, inglês e espanhol. Quanto ao idioma, 19 publicações encontram-se apenas em português, 12 estão simultaneamente em português e inglês, nove somente em inglês e um tem tradução para os três idiomas.

Cerca de três quartos dos autores são do sexo feminino. Foram identificados 165 autores, perfazendo a média de quatro por publicação, sendo que um dos textos teve dez. Não é possível perceber autores ou centros especializados na interface atenção pós-parto e avaliação de programas, uma vez que há grande dispersão nas produções no que se refere à autoria. Entretanto, é possível afirmar que os oito autores, com mais de um trabalho, têm em comum a produção de pesquisas no âmbito da pós-graduação stricto sensu sobre saúde da mulher, com base em informações disponíveis nos respectivos currículos Lattes (CNPq).

Os autores com mais de um artigo são: Bethsáida de Abreu Soares Schmitz ${ }^{\mathbf{2 3}, \mathbf{4 1}}$ (professora doutora vinculada à Universidade de Brasília, tem produção sobre o tema avaliação de políticas públicas e programas de alimentação e nutrição); Carla Betina Andreucci26,35 (professora doutora da Universidade Federal de São Carlos, tem experiência na área de medicina, com ênfase em saúde materno-infantil e epidemiologia, foi orientada no mestrado e doutorado por José Guilherme Cecatti); Cristina Maria Garcia de Lima Parada ${ }^{29,43}$ (professora doutora da Universidade Estadual Paulista Júlio de Mesquita Filho - Botucatu, atua na área de Enfermagem Obstétrica, tendo experiência em avaliação de serviços de saúde); Escolástica Rejane Carneiro Moura (professora doutora da Universidade Federal do Ceará, desenvolve pesquisas na área de saúde sexual e reprodutiva, com produção vinculada à Linha de Pesquisa Enfermagem e Políticas e Práticas de Saúde) ${ }^{25,44}$; José Guilherme Cecatti24,26,28,35,39,45 (professor doutor da Universidade Estadual de Campinas na área de Medicina, na área de Obstetrícia, tem produção sobre epidemiologia com enfoque na saúde materna e reprodutiva); Maria de Fátima Moura de Araújo ${ }^{23,41}$ (mestre, formada no programa de Nutrição Humana da Universidade de Brasília, teve Bethsáida Schmitz como orientadora de sua dissertação sobre avaliação da Iniciativa Hospital Amigo da Criança, servidora pública vinculada ao Ministério da Saúde, onde atuou como nutricionista da Área Técnica da Saúde da Mulher); Suzanne Jacob Serruya $\mathbf{2 4 , 2 8 , 4 5}$ (formada em medicina, foi professora da Universidade do Estado do Pará e consultora do Ministério da Saúde, atualmente ocupa o cargo de Assessora Regional de Saúde Sexual e Reprodutiva para as Américas da OMS/Opas (Organização Pan-Americana da Saúde), no Centro Latino Americano de Perinatologia, sua tese de doutorado sobre o PHPN foi orientada por Cecatti); Tânia Di Giacomo do Lago $\mathbf{2 4 , 2 8 , 4 5}$ (professora da Faculdade de Ciências Médicas da Santa Casa de São Paulo, foi coordenadora da Área Técnica de Saúde da Mulher do Ministério da Saúde, no período de desenvolvimento do PHPN, também integrou a Secretaria de Estado de Saúde de São Paulo). Com maior 
quantidade de trabalhos, Cecatti subscreveu seis artigos como coautor, três dos quais em parceria com Serruya e Lago. De modo que os três são os autores com maior número de publicações. Cabe destacar que cinco dos seis artigos assinados por Cecatti versam sobre o PHPN.

Considerando que Furtado e Vieirada-Silva ${ }^{1}$ apontam a formação do GT de Avaliação da Abrasco como um elemento da constituição do espaço da avaliação em saúde no Brasil, procurou-se identificar quais dos autores dos estudos analisados compõem o referido GT. Do total, somente quatro pesquisadores constam na lista fornecida no portal da Abrasco ${ }^{\mathbf{4 6}}$ como vinculados ao grupo temático. O GT de Avaliação foi constituído como fórum oficial para reunião de pesquisadores e interessados no desenvolvimento do campo do monitoramento e da avaliação em saúde no País, embora sua formação não tenha sido pacífica, devido ao posicionamento contrário daqueles que entendiam a avaliação como transversal1.

A partir da vinculação dos autores, declarada nos artigos, foram contabilizadas 54 instituições distintas. Destas, 42 são referidas em apenas um artigo cada. As mais mencionadas são: a Universidade Estadual de Campinas - Unicamp ( 8 artigos) $)^{24,26,28,35,37,39,45,}$ 47. Ministério da Saúde do Brasil (5) $23,24,28,45,48$, Fundação Oswaldo Cruz - Fiocruz (4) 27,33,48,49, Universidade Federal de São Paulo (4) 40,50,51,52 e Universidade de Brasília (3) $\mathbf{2 3 , 4 1 , 5 3}$.

A partir da verificação institucional dos autores, foram identificadas parcerias entre universidades e órgãos de gestão governamental na realização de 12 estudos ${ }^{22,23}$, $\mathbf{2 4 , 2 8 , 3 6 , 4 0 , 4 4 , 4 5 , 4 7 , 5 0 , 5 4}$; em quatro houve parceria com entidades que prestam serviços de saúde $22,35,50,53$ e, em dois, parcerias com organizações não-governamentais ${ }^{35,40}$. As demais instituições mencionadas correspondem a universidades, faculdades e institutos de pesquisa, quase todos brasileiros, exceto dois. Sem contar o Ministério da Saúde e as universidades estrangeiras, verificou-se que 17 instituições estão localizadas na região Sudeste (34,7\%), 12 no Nordeste $(24,5 \%)$, nove no Centro-Oeste (18,4\%), oito na região Sul (16,3\%) e apenas três na região Norte $(6,1 \%)$.

Quanto à unidade territorial, 26 artigos fazem avaliações de programas localizados em municípios, 10 apresentam avaliações de âmbito nacional, três de nível estadual (Ceará, Tocantins e Goiás) e três regionais (destes, dois referem-se à Regional de Saúde de Botucatu e um à Baixada Fluminense). A distribuição dos programas avaliados por região do País - desconsiderando-se os artigos de abrangência nacional - mantém proporção similar àquela das universidades a que estão filiados os autores, isso porque se localizam na região Sudeste 13 programas avaliados (40,6\%), nove na região Nordeste $(28,1 \%)$, cinco programas no Centro-Oeste (15,6\%), três no Sul (9,4\%) e dois no Norte $(6,3 \%)$.

As pesquisas de tipo quantitativo representam $69,0 \%$ do corpus (29), as qualitativas $26,2 \%$ (11), e as que utilizaram abordagem mista $4,8 \%$ (2). Dentre a diversidade de técnicas de pesquisa empregadas, destacam-se a utilização das entrevistas (20), a análise documental (13) - registros de serviços de saúde, prontuários e carteira da gestante - e o uso de bancos de dados (11). Cabe salientar que metade dos artigos combina diferentes técnicas de coleta de dados.

A análise das palavras-chave dos artigos possibilitou a identificação dos temas que se sobressaem. As palavras foram analisadas em inglês, tendo em vista este ser o idioma comum a todos os resumos dos artigos. A figura 2 apresenta, em formato visual, os descritores presentes em no mínimo dois estudos. Quanto maior a palavra aparece representada na nuvem, mais frequente foi a sua utilização. A atenção pré-natal e gestação somam, juntas, 24 ocorrências, seguida de pós-parto (10), amamentação (10), avaliação de programas (9), parto (8), humanizar (8), saúde da mulher (8) e saúde materno-infantil (6). 
Figura 2. Nuvem de palavras formada pelos descritores dos artigos (2000-2019)

$$
\begin{aligned}
& \text { information_systems } \\
& \text { quality_of_health_care } \\
& \text { health_evaluation } \\
& \text { humanize study } \\
& \text { woman childbirth hiv human_milk } \\
& \text { mortality pregnancy } \\
& \begin{array}{c}
\text { nurse } \\
\text { brazil }
\end{array} \text { breastfeeding } \\
& \text { prenatal_care } \\
& \text { postpartum child } \\
& \text { program_evaluation homevisit } \\
& \text { womens health } \\
& \text { maternal_chiTd_health } \\
& \text { primary_health_care } \\
& \text { adōlescent health_promotion }
\end{aligned}
$$

Fonte: Elaboração própria

Quando apresentadas em forma de árvore de similitude, é possível perceber como as palavras-chave aproximam-se nos artigos, formando cinco eixos temáticos, conforme exposto na figura 3. A atenção pré-natal aparece como elo central entre todos os eixos, tecendo as conexões entre a gestação, o parto e o pós-parto. $\mathrm{O}$ eixo dos estudos sobre transmissão vertical, com destaque para o HIV, mostra-se vinculado à gestação, tendo em vista que, nessa fase, os exames para diagnóstico são fundamentais para a prevenção de doenças nas crianças. A saúde materno-infantil, relacionada ao problema da mortalidade, conecta-se ao eixo atinente ao pós-parto. Interessante notar como o eixo formado pelo período pós-parto vincula-se à atenção primária em saúde, às visitas domiciliares e ao enfermeiro. A avaliação aparece sob três designações com conexões distintas: a avaliação em saúde (health evaluation), compondo o eixo pré-natal; a avaliação de programas (program evaluation), vinculada à humanização; e simplesmente avaliação (evaluation), no eixo amamentação.

Figura 3. Árvore de similitude dos descritores dos artigos

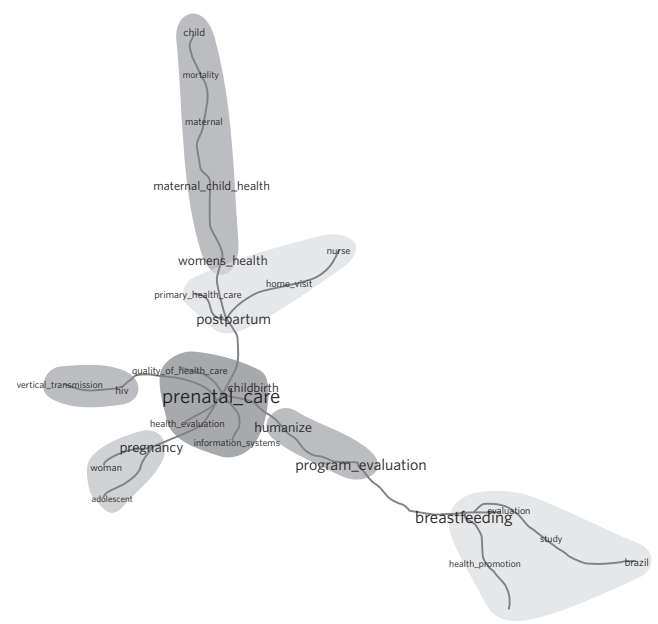


Tendo em vista a centralidade ocupada pela atenção pré-natal, o Programa de Humanização no Pré-Natal e Nascimento é o mais avaliado, com 14 estudos $\mathbf{2 4 - 2 6 , 2 8 , 2 9 , 3 4 , 3 5 , 3 7 , 3 8 , 4 3 - 4 5 , 5 0 , 5 5}$. O programa que credencia o selo de Hospital Amigo da Criança está contemplado em nove artigos $22,23,30,31,32,33,41,56,57$. A transmissão vertical da mãe para a criança é tema de três artigos com foco na prevenção do HIV 39,49,58 e um da Hepatite $B^{54}$. Três estudos analisam a Rede Cegonha ${ }^{36,48,59}$; três a visita domiciliar pós-parto $51,60,61$, dois abordam programas psicoeducativos para parentalidade ${ }^{53,62}$. Foi registrado, também, um artigo para cada um dos respectivos temas: o Sistema de Informações Hospitalares do SUS (SIH-SUS) 27; o Programa de Triagem Neonatal Auditiva (TAN) 52; o Programa Trabalhando com Parteiras ${ }^{40}$; o Programa para Suporte Integral de Adolescentes Gestantes ${ }^{\mathbf{4 2}}$; Atenção Nutricional no Pré-natal e Puerpério63; Grupo de Sala de Espera com Gestantes para prevenção da depressão puerperal47. Há, ainda, um artigo de revisão da literatura sobre programas para o pós-parto na atenção primária, que abrangeu estudos do Brasil e de outros países ${ }^{64}$.

Os documentos do Ministério da Saúde citados nos artigos foram compilados em um arquivo próprio, como estratégia para verificar as políticas, programas, orientações, manuais e boletins referenciados. O quadro 1 abaixo expõe o resultado da organização dos documentos citados estritamente relacionados às políticas e programas. Dentre os listados no quadro, o PHPN destacou-se quanto ao número de vezes em que apareceu nas referências dos artigos (16). Dos documentos complementares do Ministério da Saúde referenciados, destacam-se os manuais 'Pré-natal e puerpério: atenção qualificada e humanizada' (2006), com 11 menções; 'Parto, aborto e puerpério: assistência humanizada à mulher' (2001), com cinco; e 'Atenção ao pré-natal de baixo risco' (2012), com quatro.

Quadro 1. Políticas e programas do Ministério da Saúde do Brasil citados nas referências dos artigos analisados (2000-2019)

\begin{tabular}{ll}
\hline Ano & Nome \\
\hline 1973 & Programa Nacional de Imunizações \\
1984 & Programa de assistência integral à saúde da mulher \\
1992 & Iniciativa Hospital Amigo da Criança \\
1994 & Programa Saúde da Família \\
2000 & Programa de Humanização no Pré-Natal e Nascimento \\
2000 & Programa Trabalhando com Parteiras Tradicionais \\
2003 & Política Nacional de Humanização \\
2004 & Política Nacional de Atenção Integral à Saúde da Mulher \\
2006 & Política Nacional de Atenção Básica \\
2008 & Política Nacional de Atenção Integral à Saúde do homem \\
2009 & Programa Nacional de DST e Aids \\
2011 & Rede Cegonha \\
2001 & Política Nacional de Atenção Básica \\
2011 & Política Nacional de Alimentação e Nutrição \\
2013 & Programa Mais Médicos \\
2017 & Política Nacional de Promoção, Proteção e Apoio ao Aleitamento Materno \\
\hline
\end{tabular}

Fonte: Elaboração própria. 
Ao agrupar todos os documentos de organismos internacionais citados, as duas principais instituições mencionadas foram a OMS e o Fundo das Nações Unidas para a Infância (Unicef). $\mathrm{O}$ documento mais referido, citado em cinco artigos, foi publicado pela OMS em 1998 e intitula-se 'Evidence for the ten steps to successful breastfeeding', cuja tradução para o português foi divulgada em 2001. Os textos da Unicef citados também versam, sobretudo, a respeito da Iniciativa Hospital Amigo da Criança (IHAC).

$\mathrm{Na}$ análise das referências procurou-se identificar a presença de textos conceituais e metodológicos do campo da avaliação de programas. Houve uma multiplicidade de textos brasileiros e internacionais citados, com primazia destes. $\mathrm{O}$ autor com maior número de referências foi Avedis Donabedian, com cinco menções de textos distintos. Entre os brasileiros, não houve textos ou autores presentes em mais de um artigo.

\section{Discussão}

Dentre os 42 artigos analisados, 25 estudos foram publicados de 2010 a 2019. A distribuição temporal dos artigos é congruente com a formação dos grupos de pesquisa de avaliação em saúde descrita por Cruz, Oliveira e Campos ${ }^{3}$. As autoras identificaram a constituição de 355 grupos de pesquisa no período de 1976 a 2017. Destes, 42 grupos foram formados até 1999 e 313 a partir do ano 2000. Os anos de 2013 e 2014 foram aqueles em que houve maior número de grupos criados, $32 \mathrm{e}$ 40 , respectivamente.

Os grupos de pesquisa de avaliação em saúde estão majoritariamente vinculados à grande área das ciências da saúde $(83,1 \%)$ e, em menor proporção, às ciências humanas $(8,7 \%)$ e ciências sociais aplicadas $(3,7 \%)^{3}$. Embora a avaliação seja transversal a várias áreas de conhecimento, a predominância de grupos de pesquisa nas ciências da saúde relaciona-se ao fato de a avaliação em saúde no Brasil estar atrelada ao desenvolvimento do campo da saúde coletiva ${ }^{1,3}$. Situação verificada também nos periódicos responsáveis pela publicação dos 42 artigos analisados nesta pesquisa, todos dedicados à produção de conhecimento sobre saúde.

Das revistas que tiveram mais artigos publicados sobre programas de atenção pós-parto, a 'Cadernos de Saúde Pública' e a 'Revista de Saúde Pública' também são destacadas por Furtado e Vieira-da-Silva'. Os autores indicam que as duas revistas, juntamente com a 'Ciência e Saúde Coletiva', veicularam 287 dos 835 artigos de avaliação em saúde publicados de 1990 a 2006. Com quatro publicações que avaliam programas de atenção pós-parto, a 'Revista Brasileira de Saúde Materno Infantil’ tem histórico particular no que se refere à avaliação em saúde. De acordo com Felisberto e Samico ${ }^{65}$, o periódico evoluiu da 'Revista do Instituto de Medicina Integral Professor Fernando Figueira' (Imip), cuja primeira edição, intitulada 'Avaliação na Assistência Materno-Infantil', decorreu de um projeto de cooperação internacional com o Banco Interamericano para o Desenvolvimento (BID), com o intuito de promover a implementação da avaliação dos programas e serviços realizados pelo instituto. Quanto às três publicações presentes no 'Jornal de Pediatria', cabe mencionar que os artigos avaliaram o impacto da IHAC.

Ao verificarmos as trajetórias acadêmicas e profissionais dos oito autores com mais de um artigo de avaliação dos programas para atenção pós-parto, nota-se haver em comum a atuação profissional dedicada ao tema da saúde materno-infantil. Alguns dos autores têm experiência profissional tanto como docentes de universidades como gestores de políticas públicas. A articulação entre academia e gestão também foi identificada nas trajetórias de autoras de teses e dissertações sobre humanização do parto e nascimento ${ }^{66}$. Assim como nos estudos de Furtado e Vieira-da-Silva ${ }^{1}$, em que foi evidenciada a aproximação entre 'campo burocrático' e 'campo científico' na constituição do espaço da avaliação em saúde no Brasil. 
Nos resultados da distribuição das pesquisas, tanto referente à vinculação institucional dos autores, quanto em relação à circunscrição local do programa avaliado, notou-se maior concentração na região Sudeste com diferenças significativas em comparação com as demais regiões, sobretudo o Norte do País. Tais disparidades são similares aos dados colhidos por Cruz, Oliveira e Campos ${ }^{3}$ sobre os grupos de pesquisa de avaliação em saúde ( $\mathrm{SE}$ - 42,8\%, $\mathrm{NE}-23,9 \%, \mathrm{~S}-20,6 \%, \mathrm{CO}-8,5 \%, \mathrm{~N}-4,2 \%$ ), e por Bourguignon ${ }^{8}$, sobre a distribuição das teses e dissertações de humanização da assistência obstétrica e neonatal (SE - 53,3\%, NE $-20,6 \%, \mathrm{~S}-17,9 \%, \mathrm{CO}-7,1 \%, \mathrm{~N}-1,1 \%)$. A concentração de estudos e de grupos de pesquisa no Sudeste deve-se ao fato de a região possuir maior número de programas de pós-graduação, conforme dados do Sistema de Informações Georreferenciadas da Capes (Geocapes) ${ }^{67}$.

A análise das referências citadas sobre aspectos conceituais da avaliação demonstra a pouca inserção do debate teórico da área nos estudos analisados. Poucos artigos trouxeram bibliografia que abordasse os conceitos e métodos próprios da avaliação em saúde. Avedis Donabedian, autor reconhecido pelos trabalhos sobre avaliação da qualidade dos serviços de saúde, contou com apenas cinco menções num universo de 40 trabalhos. $\mathrm{Na}$ pesquisa de Furtado e Vieira-da-Silva', com 28 agentes vinculados à constituição do espaço da avaliação em saúde no Brasil, Donabedian foi um dos autores citados pela totalidade dos 28 entrevistados.

Outro desafio evidenciado pelos resultados é a realização de pesquisas mistas, que integrem aspectos qualitativos e quantitativos dos programas de atenção puerperal. Por exemplo, investigações sobre quantas mulheres brasileiras, em diferentes contextos regionais, conseguem realizar a consulta de revisão do parto e a qualidade desses serviços em atender às múltiplas necessidades de saúde das puérperas. São necessários também mais estudos qualitativos e quantitativos sobre a visita domiciliar, preconizada pelo Ministério da Saúde, na primeira semana após o nascimento; sobre a disponibilidade de serviços de apoio profissional à amamentação; identificação dos problemas psicológicos relativos ao puerpério e encaminhamento aos serviços especializados. Não foram identificados, por exemplo, estudos de avaliação sobre os sistemas de referência e contrarreferência, que permitem a interação entre a atenção básica e a atenção hospitalar ao parto e que são importantes para garantir a continuidade da assistência à mulher após o nascimento. Também não houve registro de estudo que abordasse programa para atender as mulheres puérperas que sofrem com a perda perinatal, embora a preocupação com a morbimortalidade materna e neonatal esteja presente nos artigos analisados.

\section{Conclusões}

Os resultados apresentados oferecem, portanto, subsídios no que tange ao baixo volume de artigos publicados sobre avaliação de programas voltados para atenção puerperal e a ausência de autores e/ou centros de pesquisa de referência na interface Avaliação de programas e puerpério. O pós-parto como um tema menos central dos estudos sobre atenção à saúde das mulheres durante o ciclo gravídico-puerperal, e a avaliação em saúde, área de caráter transversal e multifacetada em busca de consolidação como campo de estudo no interior da saúde coletiva.

Diante do exposto, pode-se afirmar que há potencial para explorar o arcabouço teórico-metodológico da avaliação em saúde para a produção de conhecimento sobre os programas de atenção puerperal, sobretudo porque a translação do conhecimento configura-se como um desafio constante para o trabalho científico e para os serviços de saúde em geral. Conforme Craveiro e Hartz ${ }^{68(536)}$,

Há uma lacuna em termos de mecanismos de translação dos conhecimentos das pesquisas para a tomada de decisões políticas, 
*Orcid (Open Researcher and Contributor ID). nomeadamente na área das determinantes sociais da saúde e a interligação com as políticas de formação e distribuição equitativa dos profissionais de saúde.

De modo que as parcerias entre o 'campo científico' e o 'campo burocrático' têm maior potencial de se traduzir em melhorias à saúde da população68-71. Sobretudo para que os serviços de saúde possam reconhecer e atender com qualidade às múltiplas necessidades de saúde das mulheres após o parto e diminuir as desigualdades existentes no uso desses serviços.

A revisão bibliométrica possibilitou caracterizar os estudos sobre programas de atenção pós-parto no Brasil em interface com a avaliação em saúde. Contudo, limitou-se aos trabalhos científicos veiculados nas bases pesquisadas e publicados em formato de artigo. Espera-se que a sistematização da produção científica empreendida neste estudo, ofereça um panorama sobre as avaliações de programas de atenção pós-parto no Brasil e incentive o desenvolvimento de novas pesquisas avaliativas que possam contribuir com a melhoria das condições de saúde das mulheres durante o puerpério.

\section{Colaboradoras}

Bourguignon AM (0000-0001-9293-6719)* contribuiu para concepção, planejamento, análise e interpretação dos dados, aprovação da versão final do manuscrito. Hartz Z (00000001-9780-9428)* e Moreira D (0000-00016203-0246)* contribuíram para concepção, planejamento, revisão crítica do conteúdo, aprovação da versão final do manuscrito

\section{Referências}

1. Furtado JP, Vieira-da-Silva LM. A avaliação de programas e serviços de saúde no Brasil enquanto espaço de saberes e práticas. Cad. Saúde Pública. 2014 [acesso em 2020 jun 26]; 30(12):2643-2655. Disponível em: https://www.scielosp.org/pdf/csp/2014. v30n12/2643-2655/pt.

2. Furtado JP, Campos GWS, Oda WY, et al. Planejamento e Avaliação em Saúde: entre antagonismo e colaboração. Cad. Saúde Pública. 2018 [acesso em 2020 jul 2]; 34(7):1-12. Disponível em: https://www. scielosp.org/article/csp/2018.v34n7/e00087917/\#.
3. Cruz MM, Oliveira SRA, Campos RO. Grupos de pesquisa de avaliação em saúde no Brasil: um panorama das redes colaborativas. Saúde debate. 2019 [acesso em 2020 jul 1]; 43(122):657-667. Disponível em: http://www. scielo.br/scielo.php?script=sci_arttext\&pid=S0103$-11042019000300657 \& \operatorname{lng}=\mathrm{en} \& \mathrm{nrm}=\mathrm{iso}$.

4. Bourguignon AM, Grisotti M. Concepções sobre humanização do parto e nascimento nas teses e dissertações brasileiras. Saúde soc. 2018 [acesso em 2020 jul 2]; 27(4):1230-1245. Disponível em: http://www. scielo.br/scielo.php?script=sci_arttext\&pid=S0104$-12902018000401230 \& \operatorname{lng}=\mathrm{pt}$. 
5. Bourguignon AM, Grisotti M. A emergência do discurso de humanização do parto e nascimento nas teses e dissertações brasileiras (1987-2012). Publ. UEPG Ci. Soc. Apl. 2016 [acesso em 2020 jun 20]; 24(3):345356. Disponível em: https://www.revistas2.uepg.br/ index.php/sociais/article/view/8951/5552.

6. Nobre LN, Freitas RR. A evolução da pós-graduação no Brasil: histórico, políticas e avaliação. Braz. J. Product. Engin. BJPE. 2017 [acesso em 2020 jun 27]; 3(2):18-30. Disponível em: https://periodicos. ufes.br/bjpe/article/view/v3n2_3.

7. Barreto ML. O desafio de avaliar o impacto das ciências para além da bibliometria. Rev. Saúde Pública. 2013 [acesso em 2020 jun 26]; 47(4):834-837. Disponível em: http://www.scielo.br/scielo.php?script=sci arttext\&pid=S0034-89102013000400834\&lng=pt.

8. Bourguignon AM. Humanização do parto e nascimento: os discursos construídos nas teses e dissertações brasileiras. 2014. [dissertação]. Florianópolis: Universidade Federal de Santa Catarina; 2014. [acesso em 2020 jun 20]. Disponível em: https://repositorio. ufsc.br/bitstream/handle/123456789/132746/333150. pdf? sequence $=1 \&$ isAllowed $=\mathrm{y}$.

9. Murta SG, Rodrigues AC, Rosa IO, et al. Avaliação de necessidades para a implementação de um programa de transição para a parentalidade. Psicol. Teoria e Pesq. 2011 [acesso em 2020 mar 3]; 27(3):337-346. Disponível em: https://www.scielo.br/scielo.php?script=sci arttext\&pid=S0102-37722011000300009\&lng=en\&n $\mathrm{rm}=\mathrm{iso} \& \operatorname{lng}=\mathrm{pt}$.

10. Brasil. Ministério da Saúde. Política Nacional de Atenção Integral à Saúde da Mulher: princípios e diretrizes. Brasília, DF: Ministério da Saúde; 2004.

11. Brasil. Ministério da Saúde. Pré-natal e puerpério: atenção qualificada e humanizada. Brasília, DF: Ministério da Saúde; 2005.

12. Brasil. Ministério da Saúde, Gabinete do Ministro. Portaria $n^{\circ} 569$, de $1^{\circ}$ de junho de 2000. Institui o Programa de Humanização no Pré-natal e Nascimento, no âmbito do Sistema Único de Saúde. Diário Oficial da União. 1 Jun 2000.
13. Corrêa MSM, Feliciano KVO, Pedrosa EN, et al. Women's perception concerning health care in the post-partum period: a meta-synthesis. Open J. Obst. Gynecol. 2014 [acesso em 2020 fev 11]; 4(7):416-426. Disponível em: https://www.scirp.org/journal/PaperInformation.aspx?PaperID=46496.

14. Corrêa MSM, Feliciano KVO, Pedrosa EM, et al. Acolhimento no cuidado à saúde da mulher no puerpério. Cad. Saúde Pública. 2017 [acesso em 2020 fev 10]; 33(3):1-12. Disponível em: https://www.scielo.br/pdf/ csp/v33n3/1678-4464-csp-33-03-e00136215.pdf.

15. Organização Mundial da Saúde. Who recommendations on postnatal care of the mother and the newborn. Genebra: OMS; 2013.

16. Langlois EV, Miszkurka M, Zunzunegui MZ, et al. Inequities in postnatal care in low and middle income countries: a systematic review and meta-analysis. Bull. World Health Organ. 2015 [acesso em 2019 nov 20]; 93(4):209-284. Disponível em: http://www. who.int/bulletin/volumes/93/4/14-140996/en/.

17. Arksey H, O'Malley L. Scoping studies: towards a methodological framework. IJSRM. 2007 [acesso em 2020 jan 13]; 8(1):19-32. Disponível em: https://www.tandfonline.com/doi/full/10.1080/1364557032000119616.

18. Araújo CAA. Bibliometria: evolução histórica e questões atuais. Em Questão. 2006 [acesso em 2020 jan 15]; 12(1):11-32. Disponível em: https://seer.ufrgs.br/ EmQuestao/article/view/16/5.

19. Da Silva M, Hayashi CR, Hayashi MC. Análise bibliométrica e cientométrica: desafios para especialistas que atuam no campo. InCID. 2011 [acesso em 2020 mar 30]; 2(1):110-129. Disponível em: http://www.revistas.usp.br/incid/article/view/42337.

20. Antunes AA. Como avaliar a produção científica. Rev. Col. Bras. Cir. 2015 [acesso em 2020 jul 2]; 42(supl1):17-19. Disponível em: http://www.scielo.br/scielo.php?script=sci_arttext $\&$ pid $=$ S0100 $-69912015000800017 \& \operatorname{lng}=\mathrm{pt}$.

21. Camargo BV, Justo AM. IRAMUTEQ: um software gratuito para análise de dados textuais. Temas psi- 
col. 2013 [acesso em 2020 jul 2]; 21(2):513-518. Disponível em: http://dx.doi.org/10.9788/TP2013.2-16.

22. Braun MLG, Giugliani ERJ, Soares MEM, et al. Evaluation of the Impact of the Baby-Friendly Hospital Initiative on Rates of Breastfeeding. Am J Public Health. 2003 [acesso em 2020 mar 10]; 93(8):1277-1279. Disponível em: https://www.ncbi.nlm.nih.gov/pmc/ articles/PMC1447954/.

23. Araújo MFM, Otto AFN, Schmitz BAS. Primeira avaliação do cumprimento dos "Dez Passos para o Sucesso do Aleitamento Materno" nos Hospitais Amigos da Criança do Brasil. Rev. Bras. Saúde Mater. Infant. 2003 [acesso em 2020 maio 18]; 3(4):411-419. Disponível em: http:// www.scielo.br/scielo.php?script=sci_arttext\&pid=S1519$-38292003000400006 \& \operatorname{lng}=$ en\&nrm=iso.

24. Serruya SJ, Cecatti JG, Lago TDG. Programa de Humanização no Pré-natal e Nascimento do Ministério da Saúde no Brasil: resultados iniciais. Cad. Saúde Pública. 2004 [acesso em 2020 fev 4]; 20(5):12811289. Disponível em: http://www.scielo.br/scielo. php?script=sci_arttext\&pid=S0102-311X20040005 $00022 \& \operatorname{lng}=$ en\&nrm=iso.

25. Passos AA, Moura ERF. Process indicators in the Program for Humanization of Prenatal Care and Childbirth in Ceará State, Brazil: analysis of a historical series (2001-2006). Cad. Saúde Pública. 2008 [acesso em 2020 fev 5]; 24(7):1572-1580. Disponível em: http://www.scielo.br/scielo.php?script=sci_arttext\&pid=S0102-311X20 08000700012\&lng=en\&nrm=iso.

26. Andreucci CB, Cecatti JG. Desempenho de indicadores de processo do Programa de Humanização do Pré-natal e Nascimento no Brasil: uma revisão sistemática. Cad. Saúde Pública. 2011 [acesso em 2020 fev 28]; 27(6):1053-1064. Disponível em: http://www. scielo.br/scielo.php?script=sci_arttext\&pid=S0102$-311 X 2011000600003 \& \operatorname{lng}=$ en\&nrm=iso

27. Nakamura-Pereira M, Mendes-Silva W, Dias MAB, et al. Sistema de Informações Hospitalares do Sistema Único de Saúde (SIH-SUS): uma avaliação do seu desempenho para a identificação do near miss materno. Cad. Saúde Pública. 2013 [acesso em 2020 mar 3]; 29(7):1333-1345. Disponível em: http://www. scielo.br/scielo.php?script=sci_arttext $\&$ pid $=$ S0102$-311 X 2013000700008 \& \operatorname{lng}=\mathrm{en} \& \mathrm{nrm}=$ iso.

28. Serruya SJ, Lago TDG, Cecatti JG. O panorama da atenção pré-natal no Brasil e o Programa de Humanização do Pré-natal e Nascimento. Rev. Bras. Saúde Mater. Infant. 2004 [acesso em 2020 mar 9]; 4(3):269-279. Disponível em: http://www.scielo.br/scielo.php?script $=$ sci_arttext $\&$ pid $=$ S1519$-38292004000300007 \& \operatorname{lng}=\mathrm{en} \& \mathrm{nrm}=$ iso.

29. Parada CMGL. Avaliação da assistência pré-natal e puerperal desenvolvidas em região do interior do Estado de São Paulo em 2005. Rev. Bras. Saúde Mater. Infant. 2008 [acesso em 2020 mar 16]; 8(1):113-124. Disponível em: http://www.scielo.br/scielo.php?script=sci_arttext \&pid=S1519$-38292008000100013 \& \operatorname{lng}=\mathrm{en} \& \mathrm{nrm}=$ iso.

30. Oliveira LS, Espírito Santo ACG. O processo de avaliação da Iniciativa Hospital Amigo da Criança sob o olhar dos avaliadores. Rev. Bras. Saúde Mater. Infant. 2013 [acesso em 2020 mar 12]; 13(4):297-307. Disponível em: http:// www.scielo.br/scielo.php?script=sci_arttext\&pid=S1519$-38292013000400297 \& \operatorname{lng}=$ en\&nrm=iso.

31. Coutinho SB, Lima MC, Ashworth A, et al. The impact of training based on the Baby-Friendly Hospital Initiative on breastfeeding practices in the Northeast of Brazil. J Pediatr (Rio J). 2005 [acesso em 2020 maio 19]; 81:471-477. Disponível em: http:// www.jped.com.br/conteudo/05-81-06-471/ing.pdf.

32. Caldeira AP, Gonçalves E. Assessment of the impact of implementing the Baby-Friendly Hospital Initiative. J Pediatr (Rio J). 2007 [acesso em 2020 mar 15]; 83(2):127-132. Disponível em: http://www.scielo.br/ pdf/jped/v83n2/en_v83n2a06.pdf.

33. Cardoso LO, Vicente AST, Damião JJ, et al. The impact of implementation of the Breastfeeding Friendly Primary Care Initiative on the prevalence rates of breastfeeding and causes of consultations at a basic healthcare. J Pediatr (Rio J). 2008 [acesso em 2020 maio 19]; 84(2):147-153. Disponível em: https://www. redalyc.org/pdf/3997/399738160010.pdf. 
34. Almeida CAL, Tanaka OY. Perspectiva das mulheres na avaliação do Programa de Humanização do Pré-Natal e Nascimento. Rev. Saúde Pública. 2009 [acesso em 2020 mar 16]; 43(1):98-104. Disponível em: http://www.scielo.br/scielo.php?script=sci arttext\&pid=S0034-89102009000100013\&lng=en\& nrm=iso.

35. Andreucci CB, Cecatti JG, Macchetti CE, et al. Sisprenatal como instrumento de avaliação da qualidade da assistência à gestante. Rev. Saúde Pública. 2011 [acesso em 2020 fev 5]; 45(5):854-864. Disponível em: http://www.scielo.br/scielo.php?script=sci arttext\&pid=S0034-89102011000500006\&lng=en\& nrm=iso.

36. Almeida KJQ, Roure FN, Bittencourt RJ, et al. Active health Ombudsman service: evaluation of the quality of delivery and birth care. Rev. Saúde Pública. 2018 [acesso em 202012 mar]; 52(76):1-11. Disponível em: http://www. scielo.br/scielo.php?script=sci_arttext\&pid=S0034$-89102018000100268 \& \operatorname{lng}=$ en\&nrm=iso.

37. Vilarinho LM, Nogueira LT, Nagahama EEI. Avaliação da qualidade da atenção à saúde de adolescentes no pré-natal e puerpério. Esc. Anna Nery. 2012 [acesso em 2020 mar 13]; 16(2):312-319. Disponível em: http://www.scielo.br/scielo.php?script=sci arttext\&pid=S1414-81452012000200015\&lng=en.

38. Oliveira DC, Mandú ENT, Corrêa ACP, et al. Estrutura organizacional da atenção pós-parto na estratégia saúde da família. Esc. Anna Nery. 2013 [acesso em 2020 mar 12]; 17(3):446-454. Disponível em: http://www. scielo.br/scielo.php?script=sci_arttext\&pid=S1414$-81452013000300446 \& \operatorname{lng}=e n \& n r m=i s o$.

39. Amaral E, Assis-Gomes F, Milanez H, et al. Timely implementation of interventions to reduce vertical HIV transmission: a successful experience in Brazil. Rev Panam Salud Publica/Pan Am J Public Health. 2007 [acesso em 2020 mar 3]; 21(6). Disponível em: https://www.scielosp.org/article/rpsp/2007. v21n6/357-364/\#ModalArticles.

40. Gusman CR, Viana APAL, Miranda MAB, et al. Inclusão de parteiras tradicionais no Sistema Único de Saúde no Brasil: reflexão sobre desafios. Rev Panam Salud Publica/Pan Am J Public Health. 2015 [acesso em 2020 fev 5]; 37(4/5):365-70. Disponível em: https://www.scielosp.org/pdf/rpsp/2015.v37n45/365-370/pt.

41. Araújo MFM, Schmitz BAS. Reassessment of Baby-friendly Hospitals in Brazil. J Hum Lact. 2007 [acesso em 2020 maio 19]; 23:246-252. Disponível em: http:// www.ibfan.org.br/documentos/outras/doc-247.pdf.

42. Sant'Anna MJC, Carvalho KAM, Melhado A, et al. Teenage pregnancy: impact of the integral attention given to the pregnant teenager and adolescent mother as a protective factor for repeat pregnancy. Scientific World. 2007 [acesso em 2020 mar 10]; (7):187-194. Disponível em: https://www.ncbi.nlm.nih.gov/pmc/ articles/PMC5901305/pdf/TSWJ-2007-7-438074.pdf.

43. Parada CMGL, Tonete VLP. Healthcare during the pregnancy-puerperium cycle from the perspective of public service users. Interface (Botucatu). 2008 [acesso em 2020 fev 5]; 12(24):35-46. Disponível em: http://www.scielo.br/scielo.php?script=sci arttext\&pid=S1414-32832008000100004\&lng=en \& nrm=iso.

44. Grangeiro GR, Diógenes MAR, Moura ERC. Atenção Pré-Natal no Município de Quixadá-CE segundo indicadores de processo do Sisprenatal. Rev. esc. enferm. USP. 2008 [acesso em 2021 abr 7]; 42(1):105-111. Disponível em: http://www.scielo.br/scielo.php?script=sci arttext\&pid=S0080-62342008000100014\&lng=en\& nrm=iso.

45. Serruya SJ, Lago TDG, Cecatti JG. Avaliação preliminar do programa de humanização no pré-natal e nascimento no Brasil. Rev. Bras. Ginecol. Obstet. 2004 [acesso em 2020 mar 9]; 26(7):517-525. Disponível em: http://www.scielo.br/scielo.php?script=sci arttext\&pid=S0100-72032004000700003\&lng=en\& nrm=iso.

46. Associação Brasileira de Saúde Coletiva. Grupo Temático de Monitoramento e Avaliação de Programas, Serviços, Sistemas e Políticas de Saúde - GT de Avaliação. Composição. Rio de Janeiro: Abrasco; 2020. 
[acesso em 2021 set 8]. Disponível em: https://www. abrasco.org.br/site/gtmonitoramentoeavaliacaodeprogramasepoliticasdesaude/composicao/.

47. Fragelli CMB, Salomão FG, Vazquez FL, et al. Eficiência de um grupo de sala de espera na adesão do aleitamento materno exclusivo. Odonto. 2011 [acesso em 2020 fev 5]; 19(38):123-129. Disponível em: https:/www.metodista.br/revistas/revistas-metodista/ index.php/Odonto/article/view/2523/0.

48. Cavalcanti PCS, Gurgel Junior GD, Vaconcelos ALR, et al. Um modelo lógico da Rede Cegonha. Physis. 2013 [acesso em 2020 jul 3]; 23(4):1297-1316. Disponível em: http://www.scielo.br/scielo.php?script=sci arttext\&pid=S0103-73312013000400014\&lng=en .

49. Araújo ESP, Friedman RK, Camacho LAB, et al. Cascade of access to interventions to prevent HIV mother to child transmission in the metropolitan area of Rio de Janeiro, Brazil. Braz J Infect Dis. 2014 [acesso em 2020 fev 5]; 18(3):252-260. Disponível em: http:// www.scielo.br/scielo.php?script=sci arttext\&pid=S1413$-86702014000300252 \& \operatorname{lng}=e n \& n r m=$ iso.

50. Succi RCM, Figueiredo EM, Zanatta LC, et al. Avaliação da assistência pré-natal em unidades básicas do município de São Paulo. Rev Latino-am Enfermagem. 2008 [acesso em 2021 abr 7]; 16(6):1-8. Disponível em: https:// www.scielo.br/pdf/rlae/v16n6/pt_08.pdf.

51. Silva LLB, Feliciano KVO, Oliveira LNFP, et al. Cuidados prestados à mulher na visita domiciliar da "Primeira Semana de Saúde Integral”. Rev Gaúcha Enferm. 2016 [acesso em 2020 mar 4]; 37(3):19. Disponível em: https://www.scielo.br/pdf/rgenf/ v37n3/0102-6933-rgenf-1983-144720160359248.pdf.

52. Mahl FD, Mattiazzi AL, Angst OVM, et al. Estudo do perfil sociodemográfico e psicossocial de mães de neonatos e seus conhecimentos sobre a Triagem Auditiva Neonatal. O Mundo da Saúde. 2013 [acesso em 2020 fev 5]; 37(1):35-43. Disponível em: http:// www.saocamilo-sp.br/pdf/mundo_saude/101/4.pdf.

53. Murta SG, Rodrigues AC, Rosa IO, et al. Evaluation of a psycho-educational parenthood transition pro- gram. Paidéia (Ribeirão Preto). 2012 [acesso em 2020 fev 7]; 22(53):403-412. Disponível em: http://www. periodicos.usp.br/paideia/article/view/53095.

54. Perim EB, Passos ADC. Hepatite B em gestantes atendidas pelo Programa do Pré-Natal. Rev Bras Epidemiol. 2005 [acesso em 2020 fev 3]; 8(3):272-81. Disponível em: https://www.scielosp.org/article/ rbepid/2005.v8n3/272-281/\#ModalArticles.

55. Valdes INF, Santos EO, Prado EV. Programa Mais Médicos: qualificação da atenção ao pré-natal e puerpério no âmbito da Estratégia de Saúde da Família. Rev. APS. 2017 [acesso em 2020 fev 5]; 20(3):403-413. Disponível em: https://periodicos.ufjf.br/index.php/aps/ article/view/16000.

56. Souza MFL, Ortiz PN, Soares PL, et al. Avaliação da promoção do aleitamento materno em Hospitais Amigos da Criança. Rev. paul. pediatr. 2011 [acesso em 2020 fev 5]; 29(4):502-508. Disponível em: http://www.scielo.br/scielo.php?script=sci arttext\&pid=S0103-05822011000400006\&lng=en\& nrm=iso.

57. Sampaio ARR, Bousquat A, Barro C. Contato pele a pele ao nascer: um desafio para a promoção do aleitamento materno em maternidade pública no Nordeste brasileiro com o título de Hospital Amigo da Criança. Epidemiol. Serv. Saúde. 2016 [acesso em 2020 fev 5]; 25(2):281-290. Disponível em: https://www.scielosp. org/article/ress/2016.v25n2/281-290/\#.

58. Lemos LMD, Rocha TFS, Conceição MV, et al. Evaluation of preventive measures for mother-to-child transmission of HIV in Aracaju, State of Sergipe, Brazil. Rev. Soc. Bras. Med. Trop. 2012 [acesso em 2020 mar 9]; 45(6):682-686. Disponível em: http://www. scielo.br/scielo.php?script=sci_arttext\&pid=S0037$-86822012000600005 \& \operatorname{lng}=\mathrm{en} \& \mathrm{nrm}=\mathrm{iso}$.

59. Assis TR, Chagas VO, Góes RM, et al. Implementação da Rede Cegonha em uma Regional de Saúde do estado de Goiás: o que os indicadores de saúde mostram sobre atenção materno-infantil? Reciis - Rev Eletron Comun Inf Inov Saúde. 2019 [acesso em 2020 fev 5]; 13(4):843-53. Disponível em: https://www.reciis.icict. 
fiocruz.br/index.php/reciis/article/view/1595/2326.

60. Bernardi MC, Carraro TE. Poder vital de puérperas durante o cuidado de enfermagem no domicílio. Texto contexto - enferm. 2014 [acesso em 2020 mar 4]; 23(1):142-150. Disponível em: http://www. scielo.br/scielo.php?script=sci_arttext\&pid=S0104$-07072014000100142 \& \operatorname{lng}=$ en\&nrm=iso.

61. Mazzo MHSN, Brito RS, Santos FAPS. Atividades do enfermeiro durante a visita domiciliar pós-parto. Rev enferm UERJ. 2014 [acesso em 2020 fev 5]; 22(5):663-7. Disponível em: http://www.facenf.uerj. br/v22n5/v22n5a13.pdf.

62. Arrais AR, Mourão MA, Fragalle B. O pré-natal psicológico como programa de prevenção à depressão pós-parto. Saúde soc. 2014 [acesso em 2020 fev 5]; 23(1):251-264. Disponível em: http://www.scielo.br/scielo.php?script=sci_arttext $\&$ pid $=$ S0104 $-12902014000100251 \& \operatorname{lng}=$ en $\&$ nrm=iso.

63. Laporte Pinfildi ASC, Zangirolani LTO, Spina N, et al. Atenção nutricional no pré-natal e no puerpério: percepção dos gestores da Atenção Básica à Saúde. Rev. Nutr. 2016 [acesso em 2020 mar 19]; 29(1):109-123. Disponível em: http://www.scielo.br/scielo.php?script $=$ sci_arttext\&pid $=$ S1415$-52732016000100109 \& \operatorname{lng}=\mathrm{en} \& \mathrm{nrm}=$ iso.

64. Baratieri T, Natal S. Ações do programa de puerpério na atenção primária: uma revisão integrativa. Ciênc. Saúde Colet. 2019 [acesso em 2020 fev 5]; 24(11):4227-4238. Disponível em: http://www. scielo.br/scielo.php?script=sci_arttext\&pid=S1413$-81232019001104227 \& \operatorname{lng}=$ en\&nrm=iso.

65. Felisberto E, Samico I. I Encontro Luso-Brasileiro de Avaliação em Saúde e Gestão do Conhecimento: Papel do IMIP e destaques temáticos. Editorial convidado. An Inst Hig Med Trop. 2016 [acesso em 2020 jun 20]; 15(supl2):S7-S8. Disponível em: http://ihmtweb. ihmt.unl.pt/PublicacoesFB/Anais/Anais2016-suplemento2/files/assets/basic-html/page-7.html\#.
66. Bourguignon AM, Grisotti M. A humanização do parto e nascimento no Brasil nas trajetórias de suas pesquisadoras. Hist. Cienc. Saúde-Manguinhos. 2020 [acesso em 2020 jul 20]; 27(2):485-502. Disponível em: https://www.scielo.br/j/hcsm/a/zZddht4v88Y 6Vz84frYyj7Q/?format=pdf\&lang=pt.

67. Coordenação de Aperfeiçoamento de Pessoal de Nível Superior. Geocapes - Sistema de Informações Georreferenciadas. Brasília, DF: Capes; 2020. [acesso em 2020 jun 30]. Disponível em: https://geocapes.capes. gov.br/geocapes/\#.

68. Craveiro I, Hartz Z. Avaliação do papel das redes de investigação na translação do conhecimento. An Inst Hig MedTrop. 2016 [acesso em 2020 jun 20]; 15(supl2):S35-S40. Disponível em: http://ihmtweb. ihmt.unl.pt/PublicacoesFB/Anais/Anais2016-suplemento2/files/assets/basic-html/page-35.html.

69. Hartz ZMA. Prefácio. In: Santos EM, Cruz MM, organizadores. Avaliação em saúde: dos modelos teóricos à prática da avaliação de programas de controle e processos endêmicos. Rio de Janeiro: Editora Fiocruz; 2014. p. 11-14.

70. Champagne F, Contandriopoulos AP, Tanon A. Utilizar a Avaliação. In: Brousselle A, Champagne F, Contandriopoulos AP, et al. Avaliação: conceitos e métodos. 2. impr. Rio de Janeiro: Editora Fiocruz; 2011. p. 241-261.

71. Bezerra LCA, Felisberto E, Costa JMBS, et al. Translação do Conhecimento na qualificação da gestão da Vigilância em Saúde: contribuição dos estudos avaliativos de pós-graduação. Physis. 2019 [acesso em 2020 jul 3]; 29(1):1-33. Disponível em: https://scielosp.org/pdf/physis/2019.v29n1/e290112/pt.

Recebido em 20/02/2021

Aprovado em 4/08/2021

Conflito de interesses: inexistente

Suporte financeiro: Coordenação de Aperfeiçoamento de Pessoal

de Nível Superior (Capes) - Código de financiamento 001 